\title{
Observations of the radio emission field around the $\gamma$-ray source 2EG J1834-2138
}

\author{
J.A. Combi ${ }^{1,2}$ and G.E. Romero ${ }^{3}$ \\ 1 Instituto Argentino de Radioastronomía, C.C.5, (1894) Villa Elisa, Bs. As., Argentina \\ 2 Theoretical Physics Lab., Department of Physics, University of La Plata, C.C. 67, 1900 La Plata, Argentina \\ 3 Instituto Astronômico e Geofísico, Universidade de São Paulo, Av. M. Stefano 4200, CEP 043010-904 São Paulo SP, Brazil
}

Received July 2; accepted August 4, 1997

\begin{abstract}
We present results of a study of the radio emission field around the best estimate position of the lowlatitude EGRET source 2EG J1834 - 2138. The identification of this $\gamma$-ray source with the gravitational lensed AGN PKS 1830 - 211 has been recently proposed by Mattox et al. (1997). Additional support for this identification is provided here. Contamination produced by the diffuse disk emission has been removed from new radio images of the surrounding region of 2EG J1834-2138 allowing a determination of the fine radio structure. Several previously unnoticed supernova remnants have been found within a few degrees from the $\gamma$-ray source. However, the only strong radio source within the $95 \%$ source location confidence contour of 2EG J1834-2138 is PKS 1830-211. In addition, both spectrum and variability analysis of the EGRET data support the identification of both sources.
\end{abstract}

Key words: gamma rays: observations - radio continuum: galaxies — galaxies: active - galaxies: quasars: PKS $1830-211$

\section{Introduction}

The second Energetic Gamma Ray Experiment Telescope (EGRET) catalog (Thompson et al. 1995) contains 129 sources detected in time integrated exposures at $E>$ $100 \mathrm{MeV}$ during Phases 1 and 2 of the Compton Gamma Ray Observatory (CGRO) mission. Additional 28 sources detected in Phase 3 of the viewing program are listed by Thompson et al. (1996). A large percentage (60.5\%) of these sources remains unidentified till now. The main candidates for identifications are active galactic nuclei (AGNs), isolated pulsars, supernova remnants (SNRs), and OB star associations. Particularly, sources at low latitudes $\left(|b|<10^{\circ}\right)$ are thought to be of galactic nature

Send offprint requests to: J.A. Combi, first address
(Kanbach et al. 1996). Possible identifications of some of these sources with SNRs (Sturner \& Dermer 1995; Sturner et al. 1996; Esposito et al. 1996), pulsars (Merck et al. 1996), and star-forming regions (Kaaret \& Cottam 1996) have been recently proposed. However, most low-latitude $\gamma$-ray sources seem to have no straightforward counterpart at other wavelengths, and it has even been suggested that they could belong to a new type of astrophysical objects (e.g. Merck et al. 1996).

The main problem that hinders an effective identification of the lower frequency counterpart of low-latitude EGRET sources is the background contamination produced by the galactic disk radiation. For radio wavelengths the fine structure at $|b|<10^{\circ}$ is almost completely masked by the diffuse disk component. If this component can be efficiently removed, new identifications with previously unnoticed radio sources might be established.

In this paper we present a study of the radio surroundings of the $\gamma$-ray source $2 \mathrm{EG} \mathrm{J} 1834-2138$. This source has been rejected as a pulsar candidate due to its spectral shape by Merck et al. (1996), and none known SNR is close to its $95 \%$ EGRET confidence contour (see Green 1996, for an updated list of SNRs). There are not either nearby OB associations (Mel'nik \& Efremov 1995). Recently, Mattox et al. (1997) has found using a Bayesian analysis that 2 EG J1834-2138 can be identified with the strong flat-spectrum radio source PKS $1830-211$ with $98 \%$ confidence. This latter extragalactic object is a wellestablished gravitational lens system and its identification with 2EG J1834-2138, if conclusively confirmed, would be the first of such kind. However, the centroid of the region enclosed by the $95 \%$ confidence contour is at a galactic latitude $b=-6.22^{\circ}$, and consequently disk contamination might be hiding other possible radio counterparts of 2EG J1834-2138, like previously unnoticed SNRs in interaction with dense molecular clouds (Aharonian \& Atoyan 1996). In order to study this possibility we have made $1.42 \mathrm{GHz}$ observations of the field around 2EG J18342138 and removed the background radiation with a 
filtering technique. The results support the Mattox et al. identification of PKS $1830-211$ as the first multiple image gravitational lensed system detected by EGRET.

\section{Observations and data analysis}

The observations were carried out with a $30-\mathrm{m}$ telescope of the Instituto Argentino de Radioastronomía during March 1996. The telescope was equipped with a 1.42 $\mathrm{GHz}$-continuum receiver of single beam, corrugated, dualchannel feed. The bandwidth was $20 \mathrm{MHz}$ and the system temperature $\sim 90 \mathrm{~K}$. The HPBW of the antena is $\sim 34$ arcminutes at the observing frequency. The observations were made at night, in order to reduce the effects of changes in telescope structure due to ambient temperature fluctuations and terrestrial interfering signals, and intercalated in blanks during a variability monitoring campaign of the radio source PKS $1830-211$ (Romero et al. 1997).

A region of $\sim 6^{\circ} \times 6^{\circ}$ around the "best" position of $2 \mathrm{EG}$ J1834-2138 (see Thompson et al. 1995 and Mattox et al. 1996) was mapped by means of repeated fast $\left(10^{\circ} / \mathrm{min}\right)$ scans in declination, regularly spaced in right ascension. Each group of scans were averaged and processed by standard techniques (e.g. Combi \& Romero 1995 and references therein). The non-variable, powerful radio sources PKS 1814 - 63, PKS 1932 - 46, and PKS 2152 - 69 were observed for flux density and pointing calibration. The resulting flux density scale was fixed according to Wills (1975).

The result of these observations was the map shown (in galactic coordinates) in Fig. 1. The rms noise of this map is $\sim 30 \mathrm{mK}$. The strong contamination produced by the radiation originated in the galactic plane avoids a faithful discrimination of the fine radio features in this image. With the aim of removing this difficulty we have applied a filtering method originally developed by Sofue \& Reich (1979) and used by several authors in studies of regions close to the plane (e.g. Combi et al. 1995; Duncan et al. 1995). The map shown in Fig. 1 was convolved with a filtering Gaussian beam of HPBW $2^{\circ}$ yielding brightness temperatures $T_{0}^{1}$ and residuals $\triangle T^{1}=T-T_{0}^{1}$, where $T$ stands for the original temperatures. A new set of temperatures $T_{0}^{\prime 1}$ was computed according to $T_{0}^{\prime 1}=T-\triangle T^{1}$ for $\triangle T^{1}>0$, and $T_{0}^{\prime 1}=T$ for $\triangle T^{1}<0$. The procedure was repeated in order to generate $T_{0}^{2}, \triangle T^{2}=T-T_{0}^{2}$, and $T_{0}^{\prime 2}$, and so on. After $n=6$ iterations, when $\left|T_{0}^{n}-T_{0}^{n-1}\right|$ became smaller than the rms noise, a residual distribution $\triangle T^{n}=T-T_{0}^{n}$ was obtained. The resulting final map, where smooth emission with sizes scales larger than $2^{\circ}$ has been eliminated, is shown in Fig. 2. The original map can be recovered just by simple addition of the background component to this new map.

Several radio sources can be clearly seen in the filtered image. Just two of them have been previously detected: the gravitational lensed QSO PKS 1830 - 211

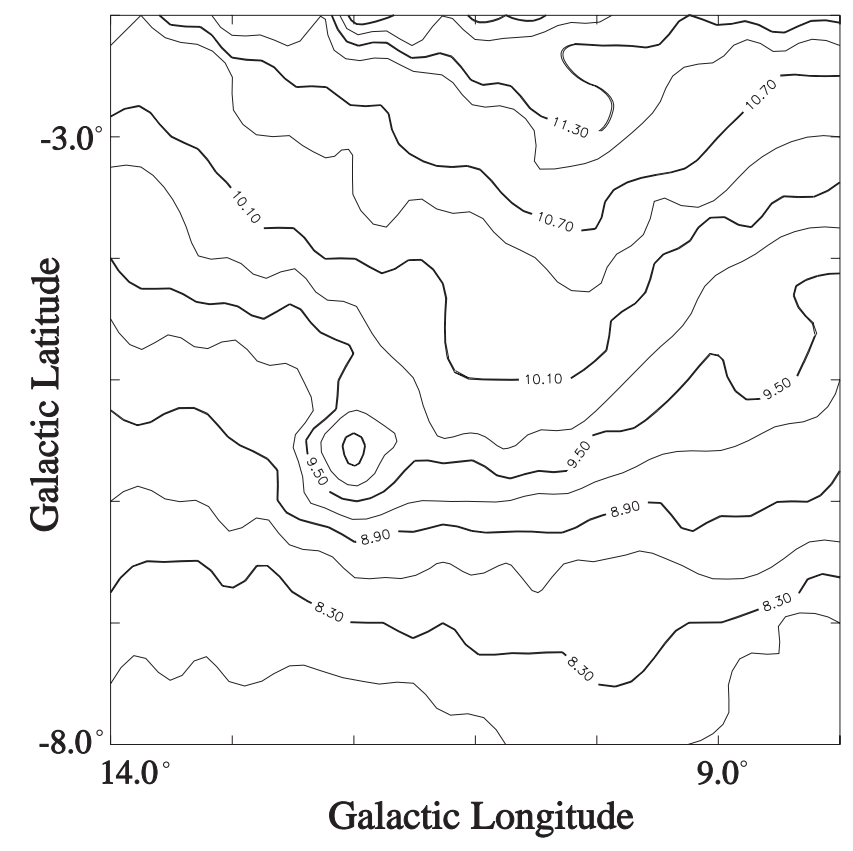

Fig. 1. Total continuum emission map at $1.42 \mathrm{GHz}$ of the region around the position of 2 EG J1834 - 2138. Contour lines are shown at $8,8.3, \ldots, 11.9 \mathrm{~K}$ in brightness temperature

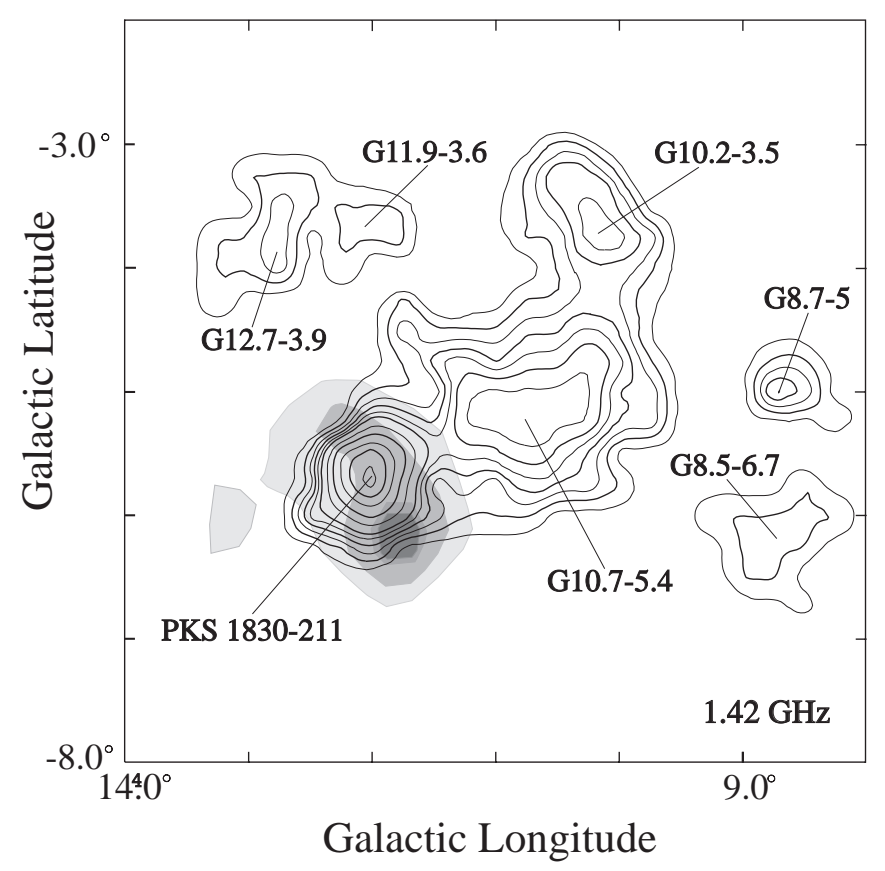

Fig. 2. The same region shown in Fig. 1 after the subtraction of the diffuse disk contribution. The confidence contours of the likelihood test statistics of 2EG J1834-2138 are also shown as a gray-scale. The contours of the radio emission are labelled in steps of $0.06,0.16, \ldots, 0.96 ; 1.3,1.6$, and $1.9 \mathrm{~K}$ in brightness temperature. The gray-scaled levels represent the $99 \%, 95 \%$, $68 \%$, and $50 \%$ statisitcal probability that the source lies within each contour 
Table 1. Main radio sources at $1.42 \mathrm{GHz}$ in the field around 2EG J1834 - 2138

\begin{tabular}{cccccc}
\hline Source & $(l, b)$ & $T_{\max }(\mathrm{K})$ & $S_{1.42 \mathrm{GHz}}(\mathrm{Jy})$ & $\alpha_{\text {mean }}$ & Id \\
\hline G8.7-5 & $(8.7,-4.9)$ & 0.51 & 1.6 & 0.1 & SNR \\
G8.5-6.7 & $(8.5,-6.7)$ & 0.36 & 1.9 & 0.21 & SNR? \\
G10.2-3.5 & $(10.2,-3.5)$ & 0.56 & 5.7 & -0.16 & SNR? \\
G10.7-5.4 & $(10.7,-5.4)$ & 0.81 & 17.7 & -0.71 & SNR \\
G11.9-3.6 & $(11.9,-3.6)$ & 0.34 & 1.1 & -0.75 & SNR \\
PKS 1830-211 & $(12.1,-5.7)$ & 1.88 & 11.8 & 0.12 & Lensed QSO \\
G12.7-3.9 & $(12.7,-3.9)$ & 0.36 & 1.9 & -0.56 & SNR \\
\hline
\end{tabular}

(Subrahmanyan et al. 1990) and the SNR G8.7 - 5.0 (Green 1996). In Table 1 we list the main characteristics of all sources in the frame.

In order to obtain some information about the nature of the new sources discovered in the field, we have used data from the $408 \mathrm{MHz}$ all-sky survey by Haslam et al. (1982) for computing spectral indices of the emission. These lower frequency data were processed in similar way than the $1.42 \mathrm{GHz}$ data and, after the removing of the background radiation, a spectral index distribution was calculated using the procedure described by Combi \& Romero $(1995,1997)$. The resulting spectral index map is shown in Fig. 3, where contour values lower than $5 \mathrm{rms}$ have been excluded. The angular resolution of this map is $\sim 50$ arcminutes due to the convolution of the $1.42-\mathrm{GHz}$ beam to the larger $408-\mathrm{MHz}$ beam. Mean errors $\left|\triangle \alpha_{\text {mean }}\right| \simeq 0.08$ have been estimated as in Combi $\&$ Romero (1997). The accuracy of the given spectral index values can be checked with PKS $1830-211$, which is a well-established flat-spectrum source (Pramesh Rao \& Subrahmanyan 1988).

\section{Results}

Except PKS 1830 - 211, all sources detected in our observations are extended. Both their morphologies and spectral indices suggest that sources G10.7 - 5.4, G11.9-3.6, and G12.7 - 3.9 are previously unnoticed SNRs. Sources G8.5 - 6.7 and G10.7 - 5.4 could be remnants with particularly flat spectra as well. The most intense of these galactic sources is G10.7 - 5.4, with an integrated flux density of $17.7 \mathrm{Jy}$ at $1.42 \mathrm{GHz}$. None known radio pulsar is associated with this object. The surface brightness is $\Sigma_{1.42 \mathrm{GHz}} \sim 3.310^{-22} \mathrm{~W} \mathrm{~m}^{-2} \mathrm{~Hz}^{-1} \mathrm{sr}^{-1}$, and its distance, according to a $\Sigma-D$ relationship (Milne 1979), would be roughly of $\sim 2.5 \mathrm{kpc}$. This implies a radius of

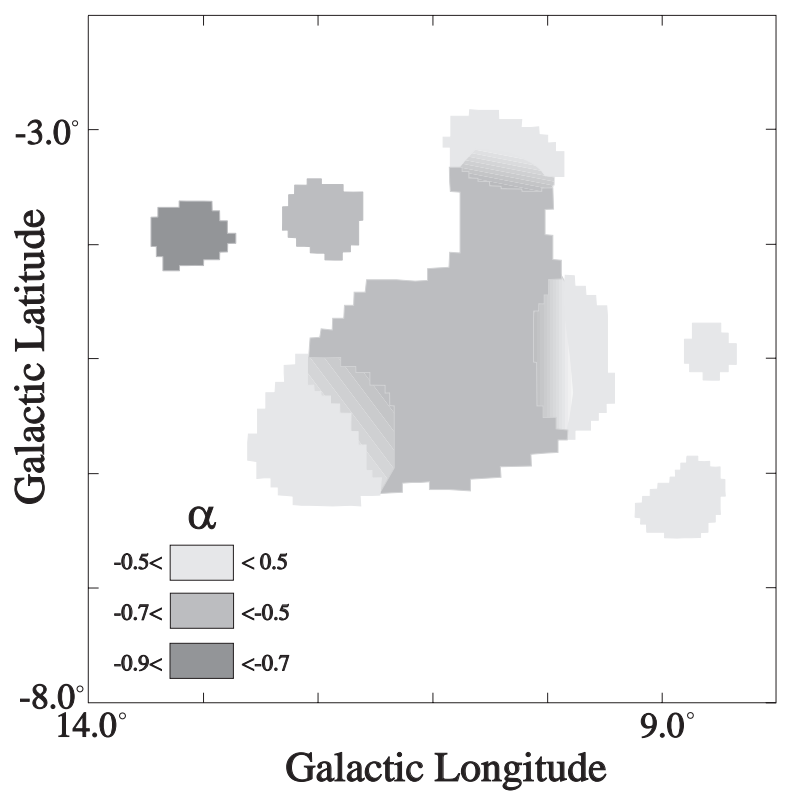

Fig. 3. Spectral index distribution computed between $408 \mathrm{MHz}$ and $1.42 \mathrm{GHz}$. The values of the spectral indices (defined as $S_{\nu} \propto \nu^{\alpha}$ ) are indicated in the map

$\sim 32.5$ pc. In a standard ISM $\left(n \sim 0.1 \mathrm{~cm}^{-3}\right)$ and assuming a Sedov phase for the expanding shell, the age of the SNR would be $\sim 4.510^{4} \mathrm{yr}$.

In Fig. 2 we have superimposed to the filtered radio map the confidence contours of the likelihood test statistics of 2EG J1834 - 2138 provided by Thompson et al. (1996). It is clear that the only source within the $95 \%$ confidence contour (which is ussually considered as representative of the EGRET statistical point source uncertainties) is PKS $1830-211$. The angular separation between the location of the highest likelihood test statistic

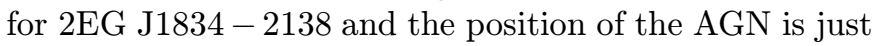


$\sim 40$ arcminutes. The remaining radio sources are too far from the EGRET source position to significantly contribute to the $\gamma$-ray emission. The nearest source is G10.7 - 5.4, and one might speculate that the pulsar produced in the supernova event that originated the remnant could be a Geminga-like object with a high proper velocity that would have driven it to the position of the EGRET detections. This seems unlikely. In fact, the angular distance from the flux peak of G10.7 - 5.4 to the EGRET highest confidence contour is $\sim 1.5^{\circ}$, which corresponds to a distance of $\sim 65 \mathrm{pc}$. The proper motion of the pulsar then should exceed $1300 \mathrm{~km} \mathrm{~s}^{-1}$, which seems unrealistic. Moreover, the pulsar hypothesis is not supported by the observed $\gamma$-spectrum of $2 \mathrm{EG}$ J1834 - 2138 (Merck et al. 1996). This spectrum can be properly fitted by a power-law given by $F(E)=(9.2 \pm$ 1.5) $10^{-10}(E / 175 \mathrm{MeV})^{-2.6 \pm 0.2} \mathrm{~cm}^{-2} \mathrm{~s}^{-1} \mathrm{MeV}^{-1}$, which is considerably steeper than observed in $\gamma$-ray pulsars (indices typically $\leq 2$, see Fierro et al. 1993). The possible presence of $\gamma$-ray variability in the time history of 2EG J1834 - 2138 (see below) also suggest to rule out a pulsar origin of the emission.

It is also worhty to remark that the Bayes's theorem has been used by Mattox et al. (1997) to computed a posteriori probability that PKS $1830-211$ is the correct iden-

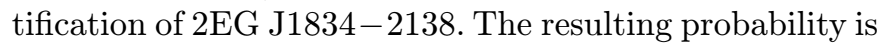
0.98 , with an a priori probability of $\sim 0.2$. In this computation radio sources from the Parkes-MIT-NRAO survey (Griffith \& Wright 1993) were used. If we take into account that there are just 183 strong flat-spectrum radio sources at $|b|>3^{\circ}$ the possibility of a chance association results negligible.

\section{Origin of the $\gamma$-ray emission}

The strong radio source PKS 1830 - 211 was first proposed to be a gravitational lensed QSO by Pramesh Rao \& Subrahmanyan (1988). High resolution radio images obtained from several interferometric arrays have revealed that the source has a ring-like structure with two bright components on sub-arcsecond scales (Jauncey et al. 1991). This suggests a close alignement of the lensed source behind the lensing object. Actually, two absorption systems have been detected at $z \simeq 0.89$ (Wiklind \& Combes 1996) and $z \simeq 0.193$ (Lovell et al. 1996), so it seems likely that the image of the background QSO (with a redshift $z \gtrsim 1$ ) is lensed by two different extragalactic objects (probably gas-rich spirals). The background source can be modeled as a core-knot-jet structure, similar to other flat-spectrum QSOs which are known to be $\gamma$-ray emitters (Nair et al.

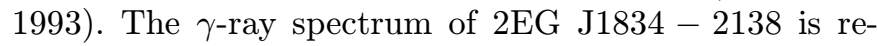
markably similar to several spectra of QSOs detected by EGRET, like $0234+285$ and $0454-463$ (von Montigny et al. 1995). These high-energy spectra are much steeper than those expected for galactic sources like pulsars. This fact, along with the spatial coincidence, strongly suggests the identification of 2EG J1834 - 2138 with PKS $1830-211$.

The presence of variability in the time history of 2EG J1834-2138 could provide additional support to the proposed identification. This time history is presented in graphical form in Fig. 4 for a $\sim 3.5$ yr time span. We have used the data from the second EGRET catalog corrected and completed by McLaughlin et al. (1996). Systematic errors over the statistical uncertainties of EGRET flux measurements are difficult to estimate. These errors can be due to uncertainties in the instrumental calibration as a function of energy, uncertainties in angle within the instrument, and errors in the galactic diffuse radiation model. McLaughlin et al. (1996) have quantified these systematic errors assuming that $\gamma$-ray pulsars are nonvariable sources, obtaining an additional uncertainty of $6.5 \% \pm 1.0 \%$ which is included in the error bars in Fig. 4.

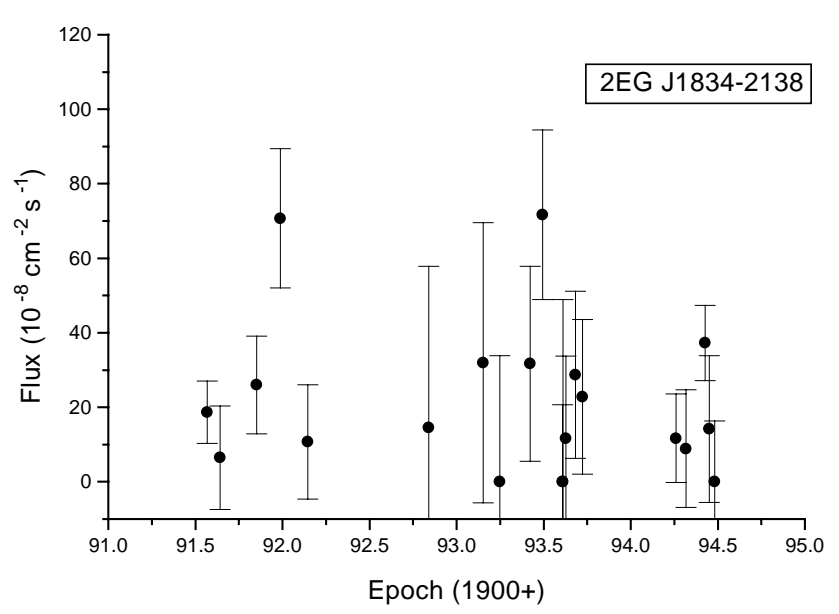

Fig. 4. Time history of $2 \mathrm{EG}$ J1834 -2138 over a period of $\sim 3.5 \mathrm{yr}$

A $\chi^{2}$-variability analysis of the entire light curve (see Romero et al. 1994) shows that the source behaviour is "probably variable". This is mainly due to the large errors in the flux values during the viewing periods from mid-1992 to mid-1993. However, if we restrict our analysis to the lapse August 1991 - February 1992 we find clear evidence for significant variability. The flux increased from $(6.5 \pm 13.9) 10^{-8} \mathrm{~cm}^{-2} \mathrm{~s}^{-1}$ in August 1991 to $(70.7 \pm 19.7) 10^{-8} \mathrm{~cm}^{-2} \mathrm{~s}^{-1}$ at the end of 1991, and then decreased to $(10.7 \pm 15.3) 10^{-8} \mathrm{~cm}^{-2} \mathrm{~s}^{-1}$ in February 1992. This implies a flux change by a factor of at least 2.5 over a time scale of $\sim 6$ months. This behaviour seems to be incompatible with a pulsar (see Ramanamurthy et al. 1995) or SNR-type source. Conversely, rapid $\gamma$-ray variability in PKS $1830-211$ could be produced both intrinsically or by gravitational microlensing. In this latter case, the background $\gamma$-ray region in the innermost part of the QSO is magnified by a compact massive object in one of the 
intervening galaxies. The variability time scale is given by the time spent by the line of sight to the source in crossing the microlens Einstein radius, i.e.

$t_{\mathrm{v}} \sim \frac{R_{\mathrm{E}}}{V} \approx 15\left(D_{\mathrm{Gpc}} M\right)^{1 / 2} v_{3}^{-1} \quad \mathrm{yr}$

where $R_{\mathrm{E}}$ is the microlens Einstein radius, $D_{\mathrm{Gpc}}=$ $D_{\mathrm{ls}} D_{\mathrm{l}} / D_{\mathrm{s}}$ is a distance in Gpc obtained from the sourcelens, lens, and source angular-diameter distances in a Robertson-Walker Universe, $M$ is the mass of the lens in units of solar masses, and $v_{3}$ is the velocity $V$ of the lens in units of $10^{3} \mathrm{~km} \mathrm{~s}^{-1}$ (see Romero et al. 1995 and references therein for details). Assuming a redshift $z_{\mathrm{s}}=1$ for the background source and $z_{1} \approx 0.89$ for the microlens, we find that $M \sim 0.02 M_{\odot}$ if $v_{3} \sim 1$ (we have considered $H_{0}=100 \mathrm{~km} \mathrm{~s}^{-1}$ and $q_{0}=1 / 2$ ). Consequently, a MACHO-like object in the halo of the foreground galaxy could produce the observed variability.

Flux variations will occur in this scenario just if the angular radius of the source in the lens plane is smaller than the Einstein angular radius of the microlens. This imposes the constraint that the size of the $\gamma$-ray emitting region should be $r \leq 1.510^{15} \mathrm{~cm} \approx 510^{-4} \mathrm{pc}$, in good accordance with the sizes expected for the $\gamma$-spheres in blazars (e.g. Blandford \& Levinson 1995). Since the $\gamma$-spheres are much smaller than the compact radio cores, no correlation with lower frequency variability should be expected for the $\gamma$-ray microlensing events. In fact, the sizes of the optical and radio emitting regions in the lens plane should largely exceed the Einstein ring sizes for small compact objects and, consequently, no significant amplifications of the lensed images should happen at these wavelengths. On the other hand, intrinsic $\gamma$-ray variability seems to occur in the initial phases of high radio outbursts (e.g. Valtaoja \& Teräsranta 1995). This fact could be used to discriminate between future intrinsic and extrinsic $\gamma$-ray variability events in PKS $1830-211$.

\section{Conclusions}

The radio field around the EGRET source 2EG J1834 2138 shows, once the galactic disk radiation has been properly subtracted, several sources, mainly SNRs. Three of these remnants are reported for first time in this paper. The only strong radio source within the $95 \%$ source location confidence contour of 2EG J1834-2138 is the flat-spectrum, gravitational lensed QSO PKS 1830 - 211 . Our observations clearly dismiss the possibility that one of the previously unknown SNRs could be the radio counterpart of the $\gamma$-ray source, strengthening the identification with the lensed blazar proposed by Mattox et al. (1997). Additional support is provided by the spectrum analy-

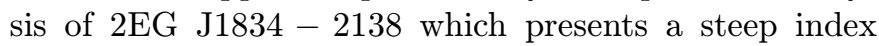
$\alpha \approx 2.6 \pm 0.2$ and by the presence of variability in the $\gamma$-ray light curve. PKS $1830-211$ seems to be, consequently, the first well-resolved gravitational lensed object detected by EGRET.

Acknowledgements. We thank E. Hurrel for assistance during the observations and an anonymous referee for call our attention to Mattox et al. (1997) paper, which was published after this work was completed. G.E.R is grateful to the IAG group of radioastronomy for assistance during the preparation of this paper. This work has been supported by the brazilian agency FAPESP and the argentine agency CONICET. Additional support from the University of La Plata (UNLP) and Grant PMTPICT 0388 from ANPCT is also gratefully acknowledged.

\section{References}

Aharonian F.A., Atoyan A.M., 1996, A\&A 309, 917

Blandford R.D., Levinson A., 1995, ApJ 441, 79

Combi J.A., Testori J.C., Romero G.E., et al., 1995, A\&A 296, 514

Combi J.A., Romero G.E., 1995, A\&A 303, 872

Combi J.A., Romero G.E., 1997, A\&AS 121, 11

Duncan A.R., Stewart R.T., Haynes R.F., et al., 1995, MNRAS 277,36

Esposito J.A., Hunter S.D., Kanbach G., et al., 1996, ApJ 461, 820

Fierro J.M., Bertsch D.L., Brazier K.T., et al., 1993, ApJ 413, L27

Green D.A., 1996, A Catalog of Galactic Supernova Remnants (Version VI), Mullard Radio Astronomy Observatory (at http://www.mrao.cam.ac.uk/surveys/snrs/)

Griffith M.R., Wright A.E., 1993, AJ 105, 1666

Haslam C.G.T., Salter C.J., Stoffel H., et al., 1982, A\&AS 47, 1

Jauncey D.L., Reynolds J.E., Tzioumis A.K., et al., 1991, Nat 352,132

Kanbach G., Bertsch D.L., Dingus B.L., et al., 1996, A\&AS 120, 461

Kaaret P., Cottam J., 1996, ApJ 462, L35

Kühr H., Witzel A., Pauliny-Toth I.I.K., et al., 1981, A\&AS 45,367

Lovell J.E.J., Reynolds J.E., Jauncey D.L., et al., 1996, ApJ $472, \mathrm{~L} 5$

McLaughlin M.A., Mattox J.R., Cordes J.M., et al., 1996, ApJ 473,763

Mattox J.R., Bertsch D.L., Chang J., et al., 1996, ApJ 461, 396

Mattox J.R., Schachter J., Molnar L., et al., 1997, ApJ 481, 95

Mel'nik A.M., Efremov Yu.N., 1995, Astron. Lett. 21, 10

Merck M., Bertsch D.L., Dingus B.L., et al., 1996, A\&AS 120, 465

Milne D.K., 1979, Aust. J. Phys. 32, 83

Nair S., Narasimha D., Pramesh Rao A., 1993, ApJ 407, 46

Pramesh Rao A., Subrahmanyan R., 1990, MNRAS 231, 229

Ramanamurthy P.V., Bertsch D.L., Fitchel C.E., et al., 1995, ApJ 450, 791

Romero G.E., Combi J.A., Colomb F.R., 1994, A\&A 288, 731 
Romero G.E., Surpi G., Vucetich H., 1995, A\&A 301, 641

Romero G.E., Benaglia P., Combi J.A., 1997, A\&AS 124, 307

Sofue Y., Reich W., 1979, A\&AS 38, 251

Sturner S.J., Dermer C.D., 1995, A\&A 293, L17

Sturner S.J., Dermer C.D., Mattox J.R., 1996, A\&AS 120, 445

Subrahmanyan R., Narashima D., Premesh Rao A., et al., 1990, MNRAS 246, 263

Thompson D.J., Bertsch D.L., Dingus B.L., et al., 1995, ApJS
101,259

Thompson D.J., Bertsch D.L., Dingus B.L., et al., 1996, ApJS 107,227

Valtaoja E., Teräsranta H., 1995, A\&A 297, L13

von Montigny C., Bertsch D.L., Chiang J., et al., 1995, ApJ 440,525

Wilkind T., Combes F., 1996, Nat 379, 139

Wills B.J., 1975, Aust. J. Phys. Suppl. 38, 1 